\title{
QUALER: A new Toolbox for Quantitative and Qualitative Analysis of Human Perceptions
}

\author{
Jose M. Alonso, David P. Pancho, L. Magdalena, D.A. Nuñez, D.S. Sánchez, P.F. Suárez ${ }^{1}$ J. Mingot, V. Iglesias $^{2}$ \\ ${ }^{1}$ European Centre for Soft Computing \\ Edificio de Investigación, Gonzalo Gutiérrez Quirós s/n, 33600 Mieres, Asturias, Spain \\ \{jose.alonso,david.perez,luis.magdalena,daniel.alvarez,daniel.sanchez,pablo.suarez\}@softcomputing.es \\ ${ }^{2}$ RØD Brand Consultants \\ Madrid, Spain \\ \{jmingot, viglesias\}@rod.com.es
}

\begin{abstract}
This paper presents a software aimed at making easier the design of customized products in accordance with consumers' expectations. It is based on a user-centered design methodology which combines techniques provided by Kansei Engineering, Sensory Analysis, and Soft Computing. Consumers' expectations are naturally expressed in the form of vague assessments related to human perceptions. Soft Computing techniques, namely Fuzzy Logic, can assist both Sensory Analysis and Kansei Engineering in the design and customization of products because of: (1) their suitability for computing with perceptions, and (2) their ability to tackle with imprecision and uncertainty in system identification. The utility of the software has been illustrated with a case study related to gin packaging.
\end{abstract}

Keywords: Computing with Perceptions, Sensory Analysis, Kansei Engineering, Soft Computing, Fuzzy Logic

\section{Introduction}

Nowadays, market differences in price and functionality among most alternatives (products/services) become almost negligible. Notice that finding out the magic formula to successfully fit the market demands is not straightforward. In fact, many new products fail because consumers' desires and needs are not clearly identified in advance [1].

The challenge resides in capturing and dealing properly with the imprecision and uncertainty inherent to consumers' opinions with respect to their satisfaction level according to tangible and intangible issues which characterize the product/service under consideration.

Alonso et al. [2] proposed to face this challenge through the combination of techniques provided by Kansei Engineering [3], Sensory Analysis [4], and Soft Computing [5]. They presented a general framework for improving the success rate of introducing new products/services into market. It is based on a novel methodology which includes four main iterative stages: (1) Prototype design; (2) Experimental setup; (3) Sensory evaluation; and (4) Intelligent data analysis. As a result, it allows to uncover and formalize the opinions of groups of people in order to guide the whole design process with the aim of minimizing the risks of failure associated to the launch of new products/services.

This paper refines the previous framework and presents a new software toolbox which implements it. The new software can be used in a wide variety of scenarios: food industry (tasting panels, consumer tests, and so on), industrial design, marketing, etc.

We have validated the utility of the new software in a practical case of use. The goal is the definition of a new packaging for a gin brand. First of all, we identified the main insights (Femininity and Originality) that should guide the design process paying attention to how they influence the whole business. To do so, we built a system dynamics model which related the main influential variables (Price, Quality, Production Costs, and so on) and potential insights (Glamour, British Personality, Femininity, Exclusiveness, Originality, etc.) given by the client specifications. Then, we set up a fuzzy web-poll, i.e. a fuzzy rating scale-based questionnaire [6] accessible online which is made up of a set of fuzzy questions with the expected responses to be expressed in fuzzy rating scales, with the aim of uncovering how consumers assess the selected insights for most commercial gin bottles. Then, we applied the fuzzy modeling methodology called HILK (abbreviation of Highly Interpretable Linguistic Knowledge) [7] in order to build fuzzy rule-based models which explain consumers' assessments. As a result, we generated two fuzzy regressors which replicate the way how consumers assessed femininity and originality of the gin bottles considered in the web-poll. Thus, those fuzzy regression models are able to automatically assess new prototypes of gin bottles, without requiring asking directly to a panel of assessors what make us save time and money. Finally, the best prototype in simulation was again evaluated and validated in another web-poll. That way, we confirmed the goodness of evaluations given by our fuzzy regression models. 
The rest of the manuscript is organized as follows. Section 2 introduces some preliminary concepts regarding Kansei Engineering, Sensory Analysis, and Soft Computing. Section 3 goes in depth with our proposal. Firstly, subsection 3.1 sketches the methodological framework. Secondly, subsection 3.2 presents the developed software. Thirdly, subsection 3.3 describes a case study which illustrates the main benefits coming out from our proposal. Finally, some conclusions and future work are pointed out in Section 4.

\section{Preliminary Concepts}

\subsection{Kansei Engineering}

The Japanese word kansei is a complex term not straightforward translated into English. Anyway, it is possible to give an informal translation referring to a kind of psychological thinking/feeling with deep roots in the Japanese culture [8].

Kansei Engineering aims at designing products/services that consumers will enjoy and will be satisfied with. To do so, human feelings and emotions (subjective consumer insights or kanseis) become the key issues which guide the design process [9, 10]. Thus, Kansei Engineering establishes a framework to formalize what consumers are expected to sense when they were exposed to new products or services $[3,9,11]$.

Human perceptions are defined by intrinsic and extrinsic attributes regarding human senses (sight, smell, taste, touch and hearing). In addition, human cognition is strongly influenced by personal background (context, mood, etc.). Human pleasantness depends on what people experience (Perceptions) and what people expect (Cognitions). In order to find out correlations between perceptions and cognitions, Kansei Engineering defines several design stages, among them we would like to highlight: (1) Selecting the right kanseis, i.e. the right insights to guide the product design; (2) selecting a pool of samples representative enough to be assessed by a panel of consumers properly chosen; (3) selecting the right statistical tests; and (4) following an iterative design based on prototypes which are sequentially evaluated and validated by the panel.

Nowadays, Kansei Engineering is known all over the world, being satisfactory applied to design products for different industries such as automotive [12], food [13] or furniture [14]. Lately, the emphasis is on the interaction with complementary methodologies and technologies [15].

\subsection{Sensory Analysis}

Sensory sciences [4] apply principles of experimental design and statistical analysis aimed at finding out consumers' likes and dislikes [16] in the evaluation of products/services [17]. Sensory data coming from human senses can be translated into valuable information for companies in order to supplement what they believe to know about consumer behavior [18].

The goal is to increase the knowledge about consumers' needs and expectations with the aim of predicting consumer responses to new products or services. To do so, sensory evaluation is carried out by panels of human assessors in charge of testing products before they are introduced into market [19].

In practice, we can distinguish between effective and affective evaluation. On the one hand, effective evaluation is related to somehow objective insights coming from panels of trained experts who are able to replicate their evaluations [17]. Of course, human perceptions are always subjective but expert panels are expected to yield almost the same assessments given close products or services. On the other hand, affective evaluation is also known as consumer testing because it deals with consumer opinions about the evaluated products/services [20]. It is usually carried out by untrained panels and therefore drawn conclusions result subjective.

\subsection{Soft Computing}

The term Soft Computing was coined in the 1990's by L. A. Zadeh [5]. Soft Computing comprises a family of complementary techniques (Fuzzy Logic, Neuro-computing, Probabilistic Reasoning, Evolutionary Computation, etc.) that cope with imprecision and uncertainty, being able to solve lots of complex real-world problems for which classical techniques cannot give accurate results [21].

Namely, fuzzy sets and systems [22] are wellknown world-wide because of their ability for linguistic concept modeling $[23,24]$. They can formalize, in an approximate but even precise way, vague concepts (like tasteful, elegant, exclusive, and so on). In addition, being recognized as universal approximators [25], fuzzy sets and systems are widely used for system identification, since they are wellsuited to carry out non-linear mappings between inputs and outputs. As a result, the use of fuzzy sets and systems lets us pass from computing with numbers to computing with perceptions [26]. Thus, it becomes natural passing from questionnaires based on the usual Likert scales to fuzzy rating scale-based questionnaires [6].

Moreover, the Computational Theory of Perceptions [27] provides a framework to implement computational systems with the capacity of computing with the meaning of linguistic expressions close to natural language, i.e. with the capacity of computing with imprecise descriptions of the world in a similar way how humans naturally do. For instance, the Highly Interpretable Linguistic Knowledge (HILK) fuzzy modeling methodology [7] is aimed at making easier the knowledge extraction and representation tasks in the context of designing interpretable fuzzy systems. It is worthy to note that the human-centric character of interpretable fuzzy systems [28] is ap- 
preciated in many applications, especially in those involving interaction with humans [29].

\section{QUALE®}

\subsection{Methodological Framework}

This section refines the framework first introduced in [2]. The goal is making easier both the product/service to market fit process and the customer relationship management process. Fig. 1 gives an overview of the whole framework which is inspired on the principles of Kansei Engineering. It follows an iterative and human-centric design approach.

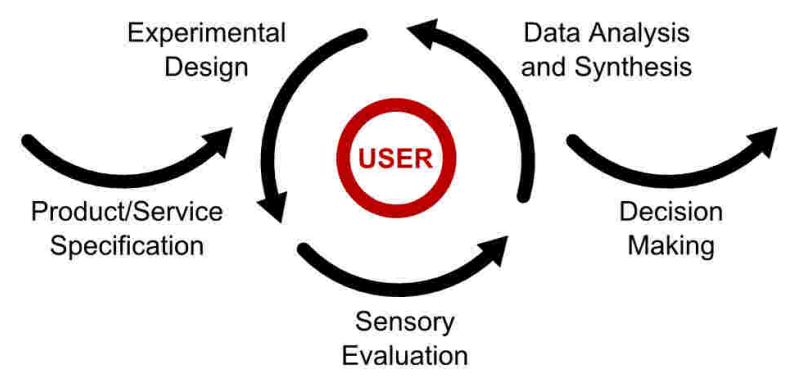

Figure 1: Methodological framework.

First of all, the person in charge of designing the new product/service must give its specification. This includes a set of desired attributes or insights (kanseis) to be satisfied. Such attributes are usually defined by vague concepts (like original, exclusive, and so on) which are naturally characterized by the fuzzy formalism. They should be determined after a careful market study (that is paying attention to customers' demands). Such study can be supported by focus groups, system dynamics software, etc. Notice that the selection of the right attributes (which are going to guide the synthesis of new products or services) is a key task deserving careful attention.

Then, the designer has to look for the best combination of design parameters (that we call labels and can be defined either numerically or linguistically depending on their nature) to satisfy the product/service specification. The right labels can be found out in an iterative process which is made up of three main stages:

1. Experimental Design. This stage establishes the statistical basis of the experimental study to be carried out in order to validate the design process. Firstly, the designer must define the hypothesis to verify along with the statistical tests to perform. Secondly, it requires an adequate selection of both number and type of (un)trained assessors who must be representative of the target market and able to recognize the attributes to evaluate for each given sample (product/service) to be tested. In addition, it is time to select carefully the attributes but also the type and number of sam- ples. Moreover, each sample must be characterized by a set of labels that refers to its physical characteristics.

2. Sensory Evaluation. It depends on the product/service (along with the set of attributes) under consideration. The evaluation is usually made through filling a questionnaire which can include several questions with different kinds of possible answers: yes/no, multiple options, checkbox, open text, ranking, rating scale, etc. Alonso et al. proposed in [2] considering the use of fuzzy rating scale-based questions, i.e. questions which are answered by means of fuzzy numbers defined in bipolar rating scales (for instance Inadequate-Adequate, WorthlessValuable, Ordinary-Unique, and so on). It is worthy to note that such fuzzy rating scales [6] can be seen as an extension of the popular Osgood's semantic differential scales [30, 31]. Thus, assessors are asked to indicate each evaluation in the form of a numerical value along with their confidence in such value. The larger the confidence interval is, the higher the imprecision and uncertainty attached to the given answer which is likely to be determined by the upper pointer but assuming that in practice it can take whatever value in the whole interval.

3. Data Analysis and Synthesis. The focus of this stage is at processing and analyzing all the answers previously collected. To do so, the designer can make use of Soft Computing techniques, mainly fuzzy logic, in order to tackle with the imprecision and uncertainty inherent to the assessors' evaluations. First of all, the goodness and reliability of the available data must be double-checked. Outliers, rare answers very far from the rest, must be identified and discarded. In addition, clustering techniques [32] can be applied in order to uncover assessor profiles according to different socio-economic criteria, but also paying attention to common preferences. It is worthy to note that all collected answers (in each group) for each sample must be properly aggregated by means of fuzzy aggregation operators [33]. Then, we deal with finding out the best matching between labels (defining the target product/service) and attributes (characterizing the customers' expectations). This task involves the use of learning algorithms, mainly fuzzy modeling $[34,35]$ and fuzzy association rule [36] learning algorithms, with the aim of understanding how assessors evaluated the given samples. The final goal is building a virtual assessor which replicates the evaluations given by human assessors. That way we can predict evaluations of unseen samples or prototypes automatically, without requiring human interaction, i.e. without requiring asking directly to the panel of assessors. This fact 
facilitates the test of new alternatives, reducing costs and speeding up the design process. Notice that, the human-centric fuzzy modeling methodology called HILK [7] is used to carry out this task in practice.

At the end of the entire process, a report for supporting decision-making is provided. It remarks in a user-friendly way (even for non-expert users) the main conclusions drawn. It makes emphasis in the degree of satisfaction or dissatisfaction of the given product/service specification by the final prototype which is expected to be ready to go to the market.

\subsection{Software Architecture}

We have developed a new software toolbox called QUALEß which implements the methodological framework just presented above. The solution covers all the stages in the framework, from the initial product/service specification to the final decision making process.

QUALE@ allows to admin several clients as well as several projects per client. In addition, it lets the user to set up and to schedule several evaluation sessions. For each session, it handles the experimental design, i.e. it assists the user in the selection of assessors, attributes, labels, samples, and so on. Notice that QUALE@ includes a wizard for designing web-polls which can include different types of questionnaires. Nowadays, the user can choose between two predefined fuzzy rating scale-based tests regarding either descriptive or comparative studies.

Once an evaluation session is closed and thus all the information from assessors is collected, QUALER assists us again regarding the statistical analysis. Later, an artificial intelligent (AI) engine is in charge of the iterative fuzzy modeling-based design of prototypes. It offers simulation and optimization capabilities that are constrained by the given product/service specification as well as by the target attributes.

The software architecture follows an MVC architectural pattern. Fig. 2 presents the modules responsible of the different components:

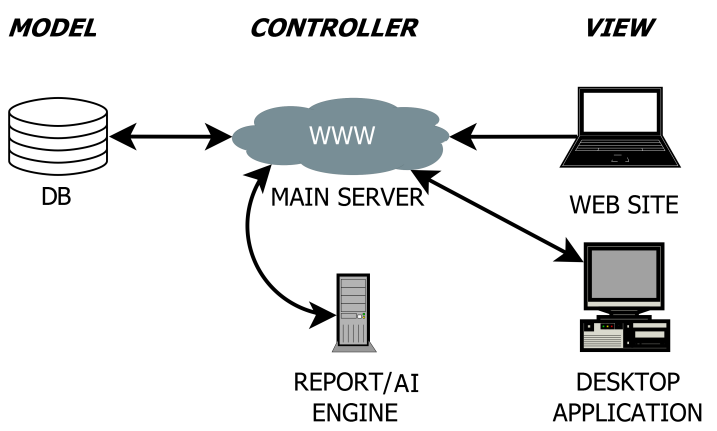

Figure 2: Software architecture.

- A Microsoft SQL Server database (DB) is responsible of the model component and contains the information of users, samples and so on. It presents persistence, ensuring data survival.

- A web server is in charge of the controller and takes a central role in the implementation. Namely, a JBoss Server runs over Linux and it hosts the web services to be accessed.

- Two front-ends:

- The desktop application permits the creation of projects, their administration and the analysis of results. It is implemented with Microsoft WPF technology that provides a visually rich environment and good interaction with the user.

- The web site houses web-polls for collecting the users' perceptions, allowing English and Spanish languages. It is implemented using HTML5, AJAX and the Javascript library Jquery. The Bootstrap framework has been used to improve the graphic details and adapt it to the use in mobile devices.

- The core of the architecture is an AI engine which is developed in $\mathrm{R}$ and Java programming languages. It is in charge of the generation of statistical reports as well as fuzzy modeling and other advanced features supported by Soft Computing techniques. This module runs in the main Server and its functionality is presented to the user through the desktop application.

\subsection{Case of Study}

The Poshmakers ${ }^{1}$, a well-recognized multi-sectorial product and services creator, proposed in 2011 the creation of the most desired premium gin brand, named Ish London Dry. The challenge was to build up a new brand from scratch, presenting a superior notoriety that favored its commercial introduction. The product specification remarked the need for notoriety but without prejudicing against its credibility and confidence in a high competitive niche. Monetary limitations constrained the quantity and quality of the labeled, packaging elements, and communication campaigns.

Summing all this up we faced a problem where the main goal was to put in place and conceive a narrative of brand that increased the value proposition and speeded its market launch up. The remainder

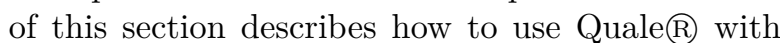
the aim of solving the problem posed above.

The first step deals with selecting the right attributes to be satisfied in accordance with the product specification. To do so, we built up a dynamic system model over the simulation platform Zio(C) $\mathrm{SD}^{2}$. That model gathers the main influential variables along with their internal causal relations.

\footnotetext{
${ }^{1}$ http://www.theposhmakers.com/

${ }^{2}$ http://www.rod.com.es/Page1.html
} 

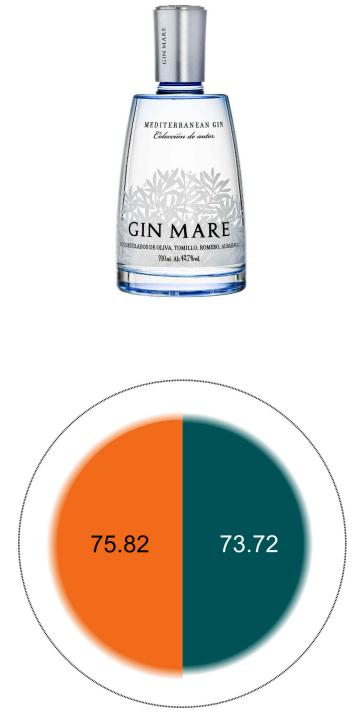
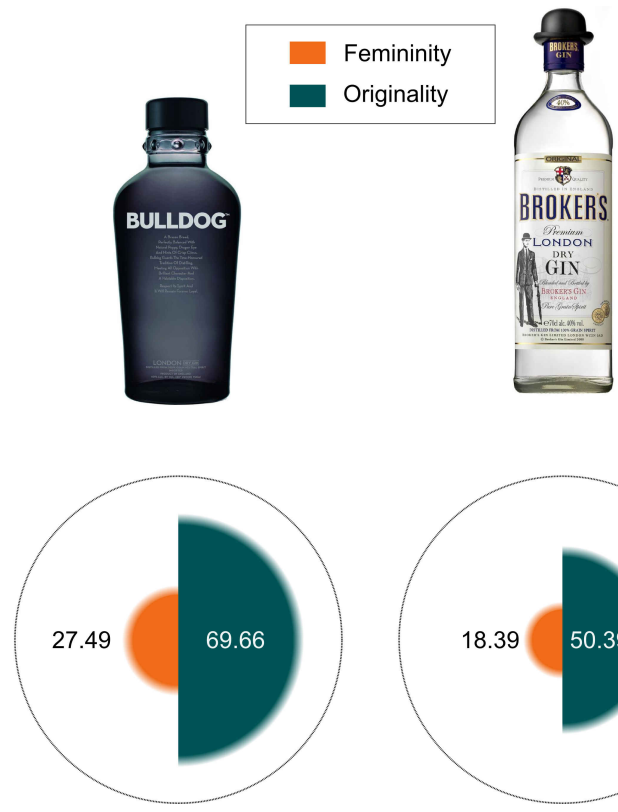
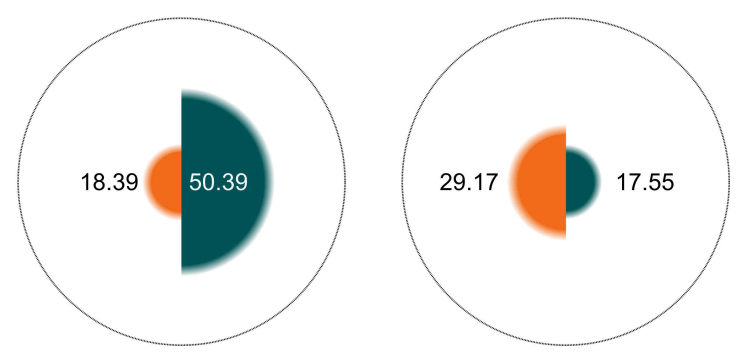

Figure 3: Results of the web-poll (Gin Mare, Bulldog, Broker's and Gordon's Gins).

After simulating and evaluating different alternative scenarios we selected Femininity and Originality as the two main attributes to be maximized in the design process.

Then, we carried out a market study which let us identify the two principal competitors (Gin Mare and Bulldog Gin) of Ish London Dry. According to our study, Gin Mare presents female features and it proposes an original design that highlights a Mediterranean style, far away from the usual London Gin concept that is popular in many others. This characteristic has reported good notoriety at the cost of a slight deterioration over time. On the contrary, we found that Bulldog Gin represents the new winner brand in the market. It is endowed with a provocative and very personalized style that turns up naturally from a gin bottle which emphasizes masculine sexuality and originality.

Thus, we faced the challenging task of positioning our brand close to Gin Mare and Bulldog Gin. With that aim, we entered in an iterative design process that conducted to build up a new gin bottle which fulfils the target requirements as detailed below.

First iteration. We collected a representative set of already commercialized gin bottles. Namely, we identified 23 different packages of some of the best well-known gin brands in the market. Notice that our selection was made with the aim of providing the pool of data samples with good heterogeneity. Each gin bottle was characterized by 9 labels regarding both Packaging (shape, weight, format, height, finishing touch and qualities) and Typography (type, style, and contrast). An expert designer was responsible for setting carefully the values related to these labels for each gin bottle under consideration.

A consumer panel made up of 50 untrained as- sessors was in charge of evaluating all the 23 samples through a fuzzy rating-based web-poll accessible online ${ }^{3}$. As a result we collected 2300 evaluations ( 23 samples $\times 2$ attributes $\times 50$ assessors) for the whole set of gin bottles. It is worthy to note that they confirmed the conclusions derived from our previous market study about Gin Mare and Bulldog Gin as it can be appreciated in Fig. 3. We have depicted with fuzzy rose diagrams [37] the aggregation of the 50 evaluations collected for each bottle. Notice that each evaluation is ranged from 0 (the center of the fuzzy rose diagram) to 100 (the external dotted blue circle). Moreover, printed values correspond to the mean of the given evaluations while the fuzzy boundaries (shadow) of the filled sectors reflect the mean confidence of assessors in their own evaluations. Thus, both Gin Mare and Bulldog Gin stand out because of their originality. In addition, Gin Mare is seen as very female (femininity scores 75.82 out of 100) while Bulldog Gin is considered as very masculine (since it gets low values of femininity). In fact, Gin Mare was the first in the ranking (regarding all the 23 bottles under study) from the point of view of femininity while Bulldog Gin was the second to last in that ranking. Gordon's Gin (one of the most classical gin brands) was the third from last in the same ranking. It is worthy to remark that only Broker's Gin was seen more masculine than Bulldog and Gordon's Gins. Gin Mare was again the first in the ranking with respect to originality, while Gordon's was the last one.

Finally, all the information previously collected was taken as training data by the AI engine of

\footnotetext{
${ }^{3}$ http://www.softcomputing.es/quale/
} 


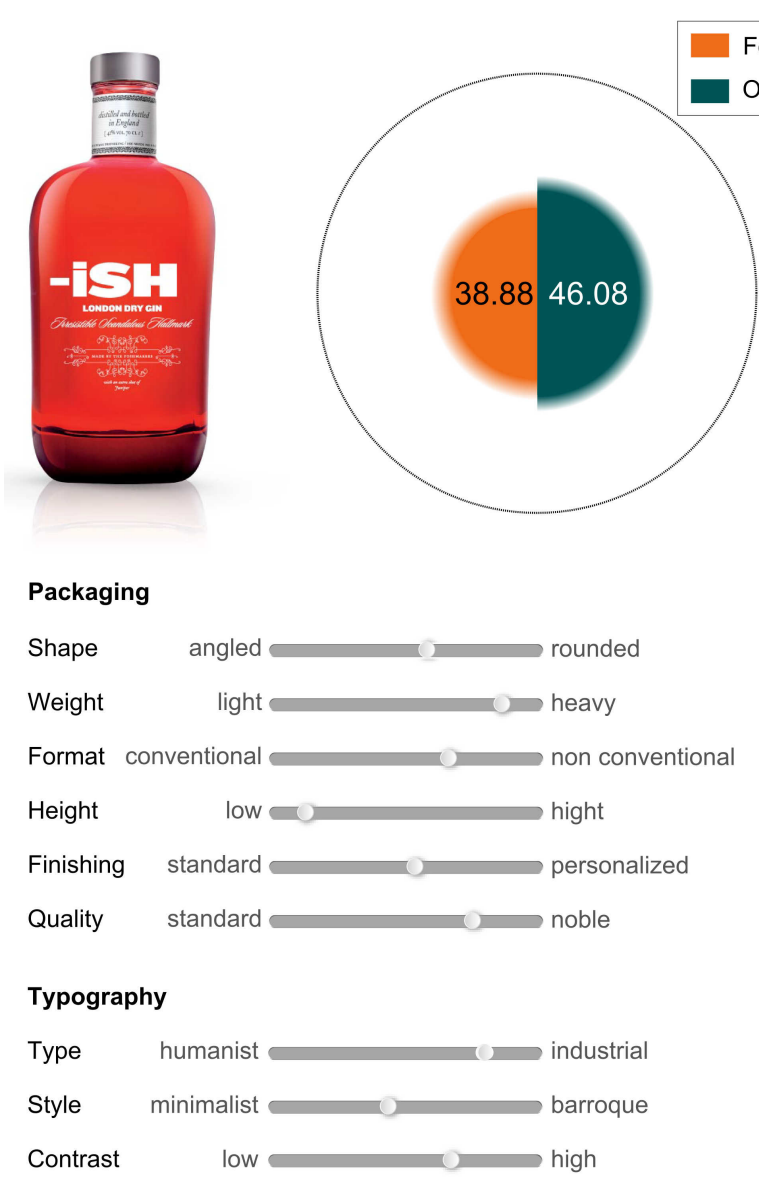

(a) First prototype

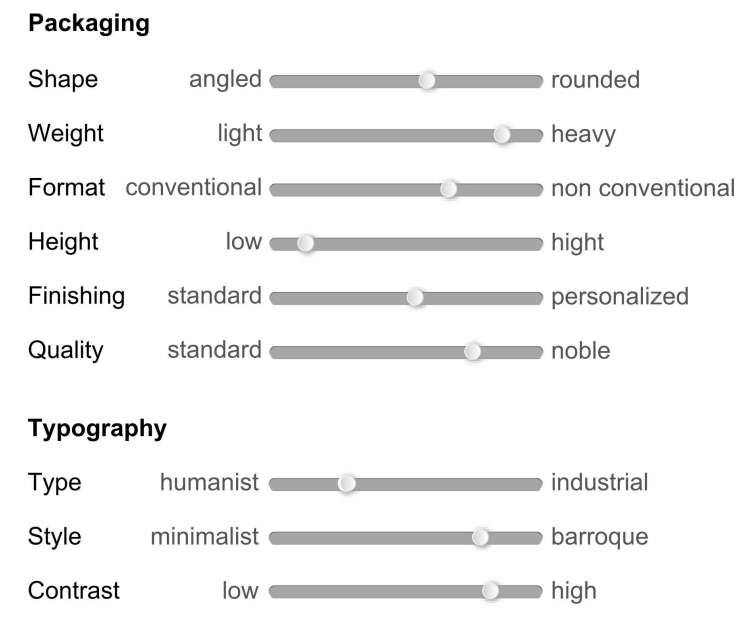

(b) Final prototype

Figure 4: Prototypes of Ish London Dry.

QUALE@ which automatically generated an AI evaluator which replicated the behavior of the group of assessors in the evaluation of the target attributes for all the given bottles. It is ready to predict the evaluations that would be given by the panel of assessors in case they were asked to evaluate new bottles. Notice that the core of this AI evaluator includes two fuzzy rule-based models (one related to femininity and the other related to originality) built by HILK methodology. Namely, we considered as rule learning algorithm the Fuzzy Decision Trees provided by GUAJE ${ }^{4}[38]$.

Second iteration. We tackled with the design of a prototype (see the top left corner of Fig. 4(a)) that, according to our background and keeping in mind the previous results, tried to maximize the two attributes of interest.

We looked for an original design by including some singular and transgressive elements. Firstly, we chose the red of the English flag of Saint George as the main color in the new bottle. This fact emphasizes the British personality of the brand. In addition, notice that there

\footnotetext{
${ }^{4}$ http://www.softcomputing.es/guaje/
}

is not any other red gin bottle in the market thus attracting the attention in the beverage showcases as a side effect. Moreover, the brand speech and iconography suggest female sexuality with a hint of fetishism and some touches of decadency and provocation.

Then, we resorted again to our expert designer who provided us with the values related to the 9 labels that characterize the new prototype in the same way how he did previously for the commercial bottles. These values are given just below the bottle in Fig. 4(a). Finally, we evaluated both femininity and originality of this prototype with the help of the AI evaluator generated at the end of the previous iteration, thus avoiding to ask directly to the panel of assessors what let us save time and money. As it can be appreciated at the top right corner of Fig. 4(a), the AI evaluator scored this prototype with low femininity (38.88) and medium originality (46.08). It is worthy to note that in this case the shadow in the fuzzy rose diagram reflects the confidence of the AI evaluator which depends on the goodness (i.e. performance measured by cross-validation) of the generated fuzzy models. 
Third iteration. We refined the previous prototype looking for a more female and original design. As it can be seen in Fig. 4(b), we kept all labels related to packaging but we changed those ones related to typography. We passed from an industrial character to a more humanistic one, with the aim of highlighting the femininity of the prototype. In addition, we passed from a minimalist style to a more baroque one, in the search for a more original prototype. We slightly increase the contrast of typography too. Then, we used again the AI evaluator in order to evaluate the goodness of the new prototype. It is easy to appreciate how the refined prototype outperforms the previous one with respect to both attributes, as desired. Femininity rises to 72.72 while originality goes up to 73.64. Notice that such evaluations are very close to those reported by Gin Mare in the first iteration of our study (see Fig. 3).

Fourth iteration. We resorted again to the panel of assessors in order to validate the goodness of the prototype achieved in the previous iteration. Assessors were asked, in another webpoll, to evaluate the selected prototype. As expected, the reported evaluations were in accordance with those provided by the AI evaluator. The prototype was scored by assessors with 68.74 to femininity and 67.96 to originality. Obviously, there are slight differences between the evaluations given by the panel and those predicted by the AI evaluator. Anyway, they are close enough to validate the goodness of the prototype which was finally selected as the packaging design of the new brand Ish London Dry. Fig. 5 shows a screenshot of QUALEß where we have included the evaluations given by the panel of assessors for some of the bottles under consideration. Ish London Dry is highlighted in red while the rest of bottles are represented by black circles.

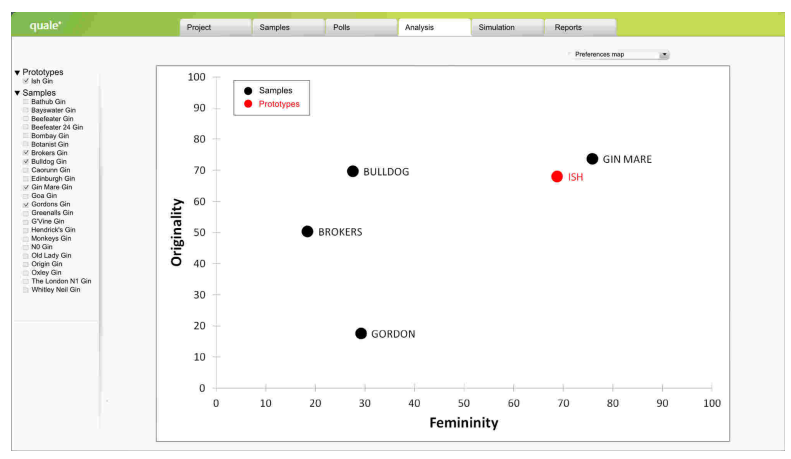

Figure 5: Screenshot of QUALE®.

\section{Conclusions}

We have presented a new software which implements a novel methodology for human-centered design of products and services. It combines techniques provided by three well-known and established research areas: Kansei Engineering, Sensory Analysis, and Soft Computing. This software has been applied to a case study about gin packaging. Notice that the brand which adopted the packaging proposed by our methodology, Ish London Dry Gin, obtained the design gold medal in 2012 Gin Masters context and its sells have tripled the initial forecasts, being out of stock the first 5 months.

In the future we will extend this software by including clustering techniques, other aggregation operators, alternative graphical representations, additional modeling and learning algorithms, etc.

\section{Acknowledgment}

This work was partially supported by the Spanish Ministry of Economy and Competitiveness under Grant TIN2014-56633-C3-3-R.

\section{References}

[1] J. B. Lord. New product failure and success. In Developing New Food Products for a Changing Marketplace, pages 4.1-4.32. CRC Press, 1999.

[2] J. M. Alonso, D. P. Pancho, and L. Magdalena. Customization of products assisted by kansei engineering, sensory analysis and soft computing. In 15th International Conference on Information Processing and Management of Uncertainty in Knowledge-Based Systems (IPMU), pages 616-625, 2014.

[3] M. Nagamachi. Kansei Engineering. Kaibundou, Tokyo, 1989.

[4] H. Stone, R. N. Bleibaum, and H. A. Thomas. Sensory evaluation practices. Food Science and Technology, International Series. Academic Press, 4th edition, 2012.

[5] L. A. Zadeh. Soft computing and fuzzy logic. IEEE Software, 11(6):48-56, 1994.

[6] S. de la Rosa de Sáa, M. A. Gil, G. GonzálezRodríguez, M. T. López, and M. A. Lubiano. Fuzzy rating scale-based questionnaires and their statistical analysis. IEEE Transactions on Fuzzy Systems, 23(1):111-126, 2014.

[7] J. M. Alonso and L. Magdalena. HILK++: an interpretability-guided fuzzy modeling methodology for learning readable and comprehensible fuzzy rule-based classifiers. Soft Computing, 15(10):1959-1980, 2010.

[8] S. Schütte. Engineering Emotional Values in Product Design. PhD thesis, Linköpings Universitet, Kansei Engineering Group, 2005.

[9] M. Nagamachi. A study of emotional technology. Japanese Journal of Ergonomics, 10(2):121-130, 1974. 
[10] M. Nagamachi. Kansei engineering: The implication and applications to product development. In IEEE International Conference on Systems, Man, and Cybernetics, pages 273278, 1999.

[11] G. Ikeda, H. Nagai, and Y. Sagara. Development of food kansei model and its application for designing tastes and flavors of green tea beverage. Food Science and Technology Research, 10(4):396-404, 2004.

[12] T. Jindo and K. Hirasago. Application studies to car interior of kansei engineering. International Journal of Industrial Ergonomics, 19(2):105-114, 1997.

[13] G. Ikeda, H. Nagai, and Y. Sagara. Development of food kansei model and its application for designing tastes and flavors of green tea beverage. Food Science and Technology Research, 10(4):396-404, 2004.

[14] M. Nagamachi. Virtual kansei engineering applied to house designing. Human Factors in Organizational Design-VI, pages 399-404, 1998.

[15] M. Nagamachi. Perspectives and the new trend of kansei/affective engineering. TQM, 20(4):290-298, 2008.

[16] M. V. Galmarini, R. Symoneaux, S. Chollet, and M. C. Zamora. Understanding apple consumers' expectations in terms of likes and dislikes. Use of comment analysis in a crosscultural study. Appetite, 62:27-36, 2013.

[17] T. Naes, P. B. Brockhoff, and O. Tomic. Statistics for Sensory and Consumer Science. Wiley, 2010.

[18] S. P. Douglas and C. S. Craig. The changing dynamic of consumer behavior. implications for cross-cultural research. International Journal of Research in Marketing, 14:379-395, 1997.

[19] C. Carter and D. Riskey. The roles of sensory research and marketing research in bringing a product to market. Food Technology, 44(11):160-162, 1990.

[20] H. G. Schutz and A. V. Cardello. Sensory Science II: Consumer Acceptance. Handbook of food science. Dekker, New York, 2003.

[21] L. Magdalena. What is Soft Computing? Revisiting possible answers. International Journal of Computational Intelligence Systems, 3(2):148-159, 2010.

[22] L. A. Zadeh. Fuzzy sets. Information and Control, 8:338-353, 1965.

[23] L. A. Zadeh. The concept of a linguistic variable and its application to approximate reasoning. Parts I, II, and III. Information Sciences, 8, 8, 9:199-249, 301-357, 43-80, 1975.

[24] E. H. Mamdani. Application of fuzzy logic to approximate reasoning using linguistic synthesis. IEEE Transactions on Computers, 26(12):1182-1191, 1977.

[25] B. Kosko. Fuzzy systems as universal approx- imators. IEEE Transactions on Computers, 43(11):1329-1333, 1994.

[26] L. A. Zadeh. From computing with numbers to computing with words - from manipulation of measurements to manipulation of perceptions. IEEE Transactions on Circuits and Systems - I: Fundamental Theory and Applications, 45(1):105-119, 1999.

[27] L. A. Zadeh. Toward a perception-based theory of probabilistic reasoning with imprecise probabilities. Journal of Statistical Planning and Inference, 105(1):233-264, 2002.

[28] W. Pedrycz. Human centricity in computing with fuzzy sets: an interpretability quest for higher order granular constructs. Journal of Ambient Intelligence and Humanized Computing, 1(1):65-74, 2010.

[29] J. M. Alonso, C. Castiello, and C. Mencar. Interpretability of fuzzy systems: Current research trends and prospects. In J. Kacprzyk and W. Pedrycz, editors, Handbook of Computational Intelligence, pages 181-199. Springer, 2014.

[30] C. E. Osgood, G. Suci, and P. Tannenbaum. The measurement of meaning. University of Illinois Press, 1957.

[31] J. G. Snider and C. E. Osgood. Semantic Differential Technique: A Sourcebook. Chicago: Aldine, 1969.

[32] J. C. Bezdek. Pattern Recognition with Fuzzy Objective Function Algorithms. Advanced Applications in Pattern Recognition. Springer, 1981.

[33] B. Bouchon-Meunier. Aggregation and Fusion of Imperfect Information. Physica-Verlag, Heidelberg, Germany, 1997.

[34] R. R. Yager and D. P. Filev. Essentials of Fuzzy Modeling and Control. New York: John Wiley, 1994.

[35] W. Pedrycz and F. Gomide. Fuzzy modeling: Principles and methodology. In Fuzzy Systems Engineering: Toward Human-Centric Computing, pages 252-275. John Wiley \& Sons, Inc., 2007.

[36] M. Delgado, N. Marín, D. Sánchez, and M.A. Vila. Fuzzy association rules: General model and applications. IEEE Transactions on Fuzzy Systems, 11(2):214-225, 2003.

[37] A. R. Buck and J. M. Keller. Visualizing uncertainty with fuzzy rose diagrams. In IEEE Symposium Series on Computational Intelligence, pages 30-36, 2014.

[38] J. M. Alonso and L. Magdalena. Generating understandable and accurate fuzzy rulebased systems in a java environment. In A. M. Fanelli, W. Pedrycz, and A. Petrosino, editors, International Workshop on Fuzzy Logic and Applications, volume 6857 of Lecture Notes in Artificial Intelligence, pages 212-219. SpringerVerlag Berlin Heidelberg, 2011. 